\title{
Metabolic associated fatty liver disease (MAFLD): a milestone in the history of fatty liver disease
}

\author{
Jawaher Alharthi, Mohammed Eslam \\ Storr Liver Centre, Westmead Institute for Medical Research, Westmead Hospital and University of Sydney, Sydney, Australia \\ Correspondence to: Mohammed Eslam. Storr Liver Centre, Westmead Institute for Medical Research, Westmead Hospital and University of Sydney, \\ Westmead 2145, Sydney, Australia. Email: mohammed.eslam@sydney.edu.au. \\ Comment on: Geier A, Tiniakos D, Denk H, et al. From the origin of NASH to the future of metabolic fatty liver disease. Gut 2021;70:1570-9.
}

Submitted Jul 06, 2021. Accepted for publication Aug 20, 2021.

doi: $10.21037 / \mathrm{hbsn}-21-269$

View this article at: https://dx.doi.org/10.21037/hbsn-21-269

With the widespread increase in energy intake and physical inactivity, the prevalence of metabolic health is declining. Consequently, metabolic associated fatty liver disease (MAFLD) [formerly known as non-alcoholic fatty liver disease (NAFLD)] has risen in prevalence and present a significant challenge to global health causing considerable morbidity and mortality, particularly when fibrosis is present (1).

MAFLD is a multisystem disorder that is heterogeneous in its underlying drivers, modifiers, spectrum of disease severity and natural history, and substantial interpatient variability across the spectrum. It is associated with an increased risk of occurring not only hepatic-related complications but also extrahepatic consequences, such as cardiovascular disease, type 2 diabetes mellitus, chronic kidney disease, and some extrahepatic cancers $(2,3)$.

The heterogeneity of the disease is likely the outcome of a complex and dynamic interaction between genetic susceptibility and multitudes of environmental factors, including age, sex, ethnicity, alcohol intake, microbiota, immune response and metabolic health, with a crucial role for epigenetic factors in mediating this interaction $(2,3)$.

The aim of this brief commentary is to highlight the recent review article written by Dr. Geier and colleagues summarising the history of fatty liver disease, particularly from a pathological perspective, which may contribute to enhancing our understanding of various aspects of the disease itself in addition to guiding future research aimed at combating the growing burden of this disease (4).

Reflecting our growing understanding of the natural history and pathogenesis of fatty liver disease and the dominant role of metabolic dysfunction in that disease, a landmark statement for the redefinition of fatty liver disease was put forth by an international expert consensus panel in 2020 to circumvent the multiple shortcomings of the previous definition $(5,6)$. This process includes the introduction of MAFLD as a more proper overarching term replacing the old term (NAFLD) and a novel set of positive diagnostic criteria for the disease substituted for its current, exclusionary definition. The diagnosis of NAFLD is based on the presence of hepatic steatosis, with the exclusion of excessive alcohol consumption based on arbitrary cut-offs and other causes of hepatic steatosis. On the contrary, the diagnosis of MAFLD can be established by the presence of hepatic steatosis and at least one of the following criteria: (I) overweight/obesity, (II) type 2 diabetes, and (III) clinical evidence of metabolic dysregulation $(5,6)$. These diagnostic criteria overcome the previous ambiguity of the negative definition of NAFLD and acknowledge that MAFLD is a stand-alone disease that can coexist with other liver diseases. Furthermore, while MAFLD avoids the stigmatisation and trivialisation accompanying NAFLD, and given the complex multifaceted nature of metabolic disorders, it brings MAFLD to the camp of other metabolic diseases, moving away from the strict and narrow hepatological-oriented view. This renders it a disease of interdisciplinary medical and health policy-making interest and holds the promise of encouraging the launching of MAFLD clinics run by multidisciplinary teams that enable proactive assessment and prompt intervention of metabolic comorbidities (2). There is an opportunity to capitalise on the management approach and facilities that are already 
being implemented in some healthcare systems for patients with other metabolic diseases.

Likely indicating that it fills a long-standing unmet need, the proposed redefinition of fatty liver disease has attracted substantial attention and has been endorsed by numerous key liver organisations, patient advocacy associations and nurse and allied health practitioners (7). More importantly, an exponentially growing number of studies have confirmed that the MAFLD criteria outperform the NAFLD criteria at multiscale, including in identifying patients with advanced hepatic fibrosis, chronic kidney disease, cardiovascular disease, higher mortality and those who would benefit from an evaluation of the GWAS identified genetic variants for fatty liver disease. This has even been reported in patients with other concomitant liver diseases, such as viral hepatitis $\mathrm{B}$ and $\mathrm{C}(8,9)$. In addition, multiple studies have demonstrated that this change from NAFLD to MAFLD helps to increase awareness of fatty liver disease among primary care physicians, specialists and patients. Furthermore, the new definition of MAFLD overcomes the previous lack of clear and easy diagnostic pathways, which can improve patients' identifications and referral pathways to further improve patient care and clinical trials.

Another aspect discussed by Dr Geier et al. (4) is the definition of non-alcoholic steatohepatitis, "NASH", which was also addressed by the redefinition proposal $(5,6)$. This acknowledges the dynamic nature of the disease and the facts that the propensity for the disease's progression can vary across lifespans and that inflammation can oscillate rapidly, even over a short period of time. It has been suggested that $\mathrm{NASH}$, as an entity, should be abandoned in favour of a more apt descriptive grading along an activity score that spans the entire MAFLD spectrum. Geier et al. (4) also touched on the genetic basis of MAFLD. Although MAFLD is typically associated with obesity and unhealthy lifestyle choices, a significant proportion of patients with MAFLD are non-obese; moreover, we know that MAFLD is a heritable polygenic disorder that likely has a shared genetic basis with other metabolic diseases. Multiple genetic variants that are implicated in disease susceptibility and progression and control key pathways in disease pathogenesis have been identified by genome-wide association studies and candidate gene studies. The growing understanding of the heritability of the disease holds the promise of pointing out novel therapeutic targets and developing a non-invasive score for disease diagnosis (10).

In conclusion, our growing understanding of the natural history and pathogenesis of MAFLD and the predominant role of metabolic dysfunction in this disease have evoked efforts to redefine fatty liver disease. Currently, efforts should be focused on capitalising on the momentum and opportunities generated by this redefinition process for improving our ability to deliver optimal multidisciplinary care by undertaking innovative clinical trials and diagnostic and interventional individualised approaches that are based on proper stratification. This will lead to improved outcomes for these patients and a decreased burden on healthcare systems.

\section{Acknowledgments}

Funding: ME is supported by the Robert W. Storr Bequest to the Sydney Medical Foundation, University of Sydney; National Health and Medical Research Council of Australia (NHMRC) Program Grants (1053206 and 1149976) and Project grants (1107178 and 1108422, 2001692).

\section{Footnote}

Provenance and Peer Review: This article was commissioned by the editorial office of Hepatobiliary Surgery and Nutrition. The article did not undergo external peer review.

Conflicts of Interest: Both authors have completed the ICMJE uniform disclosure form (available at https://hbsn. amegroups.com/article/view/10.21037/hbsn-21-269/coif). The authors have no conflicts of interest to declare.

Ethical Statement: The authors are accountable for all aspects of the work in ensuring that questions related to the accuracy or integrity of any part of the work are appropriately investigated and resolved.

Open Access Statement: This is an Open Access article distributed in accordance with the Creative Commons Attribution-NonCommercial-NoDerivs 4.0 International License (CC BY-NC-ND 4.0), which permits the noncommercial replication and distribution of the article with the strict proviso that no changes or edits are made and the original work is properly cited (including links to both the formal publication through the relevant DOI and the license). See: https://creativecommons.org/licenses/by-nc-nd/4.0/.

\section{References}

1. Eslam M, Sarin SK, Wong VW, et al. The Asian Pacific 
Association for the Study of the Liver clinical practice guidelines for the diagnosis and management of metabolic associated fatty liver disease. Hepatol Int 2020;14:889-919.

2. Eslam M, Ahmed A, Després JP, et al. Incorporating fatty liver disease in multidisciplinary care and novel clinical trial designs for patients with metabolic diseases. Lancet Gastroenterol Hepatol 2021;6:743-53.

3. Bayoumi A, Grønbæk H, George J, et al. The Epigenetic Drug Discovery Landscape for Metabolic-associated Fatty Liver Disease. Trends Genet 2020;36:429-41.

4. Geier A, Tiniakos D, Denk H, Trauner M. From the origin of NASH to the future of metabolic fatty liver disease. Gut 2021;70:1570-9.

5. Eslam M, Sanyal AJ, George J, et al. MAFLD: A Consensus-Driven Proposed Nomenclature for Metabolic Associated Fatty Liver Disease. Gastroenterology 2020;158:1999-2014.e1.

Cite this article as: Alharthi J, Eslam M. Metabolic associated fatty liver disease (MAFLD): a milestone in the history of fatty liver disease. HepatoBiliary Surg Nutr 2021;10(5):696-698. doi: 10.21037/hbsn-21-269
6. Eslam M, Newsome PN, Sarin SK, et al. A new definition for metabolic dysfunction-associated fatty liver disease: An international expert consensus statement. J Hepatol 2020;73:202-9.

7. Zheng KI, Fan JG, Shi JP, et al. From NAFLD to MAFLD: a "redefining" moment for fatty liver disease. Chin Med J (Engl) 2020;133:2271-3.

8. Fouad Y, Elwakil R, Elsahhar M, et al. The NAFLDMAFLD debate: Eminence vs evidence. Liver Int 2021;41:255-60.

9. Yamamura S, Eslam M, Kawaguchi T, et al. MAFLD identifies patients with significant hepatic fibrosis better than NAFLD. Liver Int 2020;40:3018-30.

10. Eslam M, George J. Genetic Insights for Drug Development in NAFLD. Trends Pharmacol Sci 2019;40:506-16. 